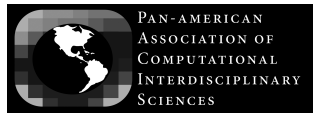

Journal of Computational Interdisciplinary Sciences (2009) 1(2): 95-98

(C) 2009 Pan-American Association of Computational Interdisciplinary Sciences

ISSN 1983-8409

http://epacis.org

\title{
Ordered structures and phase transitions in a phase field crystal model for adsorbed layers
}

\author{
Jorge A.P. Ramos ${ }^{1,2}$, Enzo Granato ${ }^{1,4}$, Cristian V. Achim ${ }^{3}$, See Chen Ying ${ }^{4}$, \\ Ken R. Elder ${ }^{5}$ and Tapio Ala-Nissila,3
}

Manuscript received on February 14, 2009 / accepted on March 10, 2009

\begin{abstract}
We study numerically the phase diagram of a phase field crystal model for adsorbed layers. This model, introduced recently, is a continuous field crystal lattice in presence of an external pinning potential. The model allows for both elastic and plastic deformations of the layer on atomic and diffusive time scales. Monte Carlo simulations are used to determine the phase diagram as a function of temperature, lattice mismatch and substrate pinning potential. The results show a rich phase diagram with commensurate, incommensurate and liquid-like phases with a topology strongly dependent on the type of ordered structure.
\end{abstract}

Keywords: adsorbed layers, commensurate-incommensurate transitions, strained epitaxial layers, numerical simulation.

\footnotetext{
Correspondence to: Jorge Anderson Paiva Ramos

${ }^{1}$ Laboratório Associado de Sensores e Materiais, Instituto Nacional de Pesquisas Espaciais, $12245-970$ São José dos Campos, SP, Brasil.

${ }^{2}$ Departamento de Ciências Exatas, Universidade Estadual do Sudoeste da Bahia, 45000-000 Vitória da Conquista, BA, Brasil.

${ }^{3}$ Laboratory of Physics, Helsinki University of Technology, P.O. Box 1100, FIN-02015 TKK, Finland.

${ }^{4}$ Department of Physics, Brown University, Providence, RI 02912-1843, USA.

${ }^{5}$ Department of Physics, Oakland University, Rochester, Michigan 48309-4487, USA.

E-mails: anderson@uesb.br / enzo@las.inpe.br / cva@cc.hut.fi / see-chenying@brown.edu / elder@oakland.edu / tapio.ala-nissila@tkk.fi
} 


\section{INTRODUCTION}

Many adsorbed systems on crystal surfaces form different ordered structures at low temperature which may be unstable against thermal fluctuations and lattice mismatch [1]. Strained epitaxial layers and commensurate layers on semiconductors [2] and metal surfaces $[1,3]$ are of particular current interest. In numerical simulations, pure elastic models or atomistic models are often employed [1]. However, there are important limitations in these approaches. In the former, plastic deformations such as dislocations are not taken into account and in the latter, the accessible time scale is very limited.

In this work, we consider the effects of thermal fluctuations and lattice mismatch in a phase field crystal model for adsorbed layers introduced recently [4]. The model describes the adsorbed layer as a continuous number density field with an external pinning potential. In the absence of pinning it reduces to the phase field crystal (PFC) model $[5,6,7]$ which has been used to model elastic and plastic deformations in crystalline materials on atomic and diffusive time scales. We use Monte Carlo (MC) simulations to determine the phase diagram as a function of temperature, lattice mismatch and substrate pinning potential.

\section{MODEL AND SIMULATION}

The free-energy of the PFC model for an adsorbed monolayer [4] can be written as

$F=F_{o} \int d^{2} \vec{r}\left\{\frac{\psi}{2}\left[r+\left(1+\nabla^{2}\right)^{2}\right] \psi+\frac{\psi^{4}}{4}+V \psi-\mu \psi\right\}$,

where $\psi(x, y)$ is a continuous field representing the number density of the adsorbed layer and $V(x, y)$ represents the pinning potential due to the substrate. The overall constant $F_{o}$ sets the energy scale. Here we have introduced an additional linear term containing a chemical potential $\mu$, which controls the variations of the field $\psi(x, y)$. This removes the conservation constraint on $\psi(x, y)$ in the original PFC model [5,6] and allows the use of a $\mathrm{MC}$ algorithm with nonconserved dynamics, which is more efficient for equilibrium simulations.

We consider a pinning potential $V(x, y)$ with a square symmetry which can represent, for example, an adsorbed layer on the (100) face of an fcc crystal [1], given by

$$
V(x, y)=V_{o}\left[\cos \left(k_{s} x\right)+\cos \left(k_{s} y\right)\right],
$$

where $k_{s}=2 \pi / a_{s}$ is the pinning potential wave vector. We define the lattice misfit between adsorbed layer and substrate as $\delta_{m}=k_{a}-k_{s}$, where $k_{a}$ is the wave vector of the hexagonal periodic pattern of $\psi(x, y)$, in the absence of the pinning potential, corresponding to a free adsorbed layer. Thus, for $\delta_{m}>0$, the layer is under tensile strain while for $\delta_{m}<0$ it is under compression. In the previous work [4], only $\delta_{m} \geq 0$ was considered.

To go beyond mean field theory and take thermal fluctuations into account, we can consider the free energy $\mathrm{F}$ of the PFC model defined above as a coarse grained effective Hamiltonian $H[\psi]$. The parameter $r$ in $H$ is taken to be a constant for the region below the mean field transition temperature $T_{m}$ in the absence of the pinning potential. The partition function is then given by $Z=\sum_{\psi} e^{-H[\psi] / k_{B} T}$ where the sum is over all configurations of $\psi(x, y)$. Other thermodynamic averages are defined in the same manner. Using this approach, we have investigated the ordered structure and phase transitions of an adsorbed layer. The averages over $\psi(x, y)$ are performed using $\mathrm{MC}$ simulations with a standard Metropolis algorithm. At each site of the discretized space we attempt to change $\psi(x, y)$ by an small amount $d \psi$ with probability $\min \left(1, e^{-d H / k_{B} T}\right)$, where $d H$ is the resulting change in the energy. Simulations were performed using typically $4 \times 10^{5} \mathrm{MC}$ steps per site for equilibration and equal number of steps for thermal averages in systems with grid size $128 \times 128$ and discretization $d x=\pi / 4$. The parameters $r=-1 / 4$ and $\mu=-0.1875$ were chosen to correspond to the crystalline region in the original PFC model without pinning $[5,6]$. Temperature is measured in units of $d x^{2} /\left(2 k_{B} F_{o}\right)$.

\section{RESULTS AND DISCUSSION}

The type of structure in the ground state was obtained by standard MC simulated annealing. The adsorbate positions are identified as the maxima in $\psi(x, y)$. Depending on the strength of the pinning potential and the misfit parameter $\delta_{m}$, three simple ordered structures were obtained as shown in Figure 1: a $(1 \times 1)$ commensurate $(\mathrm{C})$ phase, where the adsorbate lattice coincides with the pinning potential minima; a centered $(2 \times 2)$ commensurate phase (denoted as $\mathrm{c}(2 \times 2))$, where the adsorbates form a superstructure with lattice spacing twice the pinning potential spacing and an incommensurate (IC) phase, where the layer forms an hexagonal lattice or contains a structure of domain walls.

The phase diagrams near the $C$ phases $(1 \times 1)$ and $c(2 \times 2)$ were obtained by monitoring the behavior of the structure factor and specific heat as a function of temperature and lattice misfit. The structure factor was calculated as $S(k)=$ $\langle\psi(k) \psi(-k)\rangle / N$, where $\psi(k)$ is the Fourier transform of $\psi(x, y)$ and $N$ is number of grid points. The angular brackets denote thermal average. 
(1x1) phase

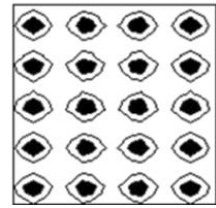

$c(2 \times 2)$ phase

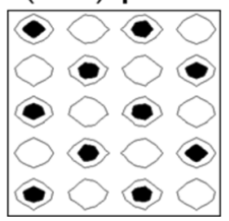

IC phase

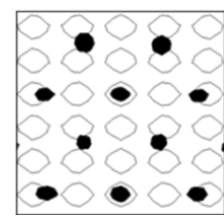

Figure 1 - Ordered structures in the ground state, depending on the amplitude of the pinning potential $V_{o}$ and misfit parameter $\delta m$. Filled circles represent the adsorbate positions and open circles correspond to the substrate pinning potential minima. Three simple structures are shown from left to right: $(1 \times 1)$ commensurate phase, $c(2 \times 2)($ centered $(2 \times 2))$ commensurate phase and incommensurate hexagonal phase (IC).

(a)

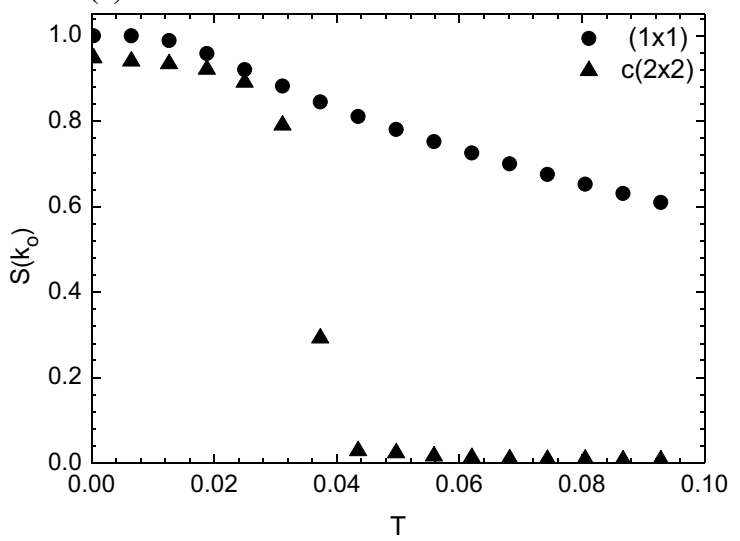

(b)

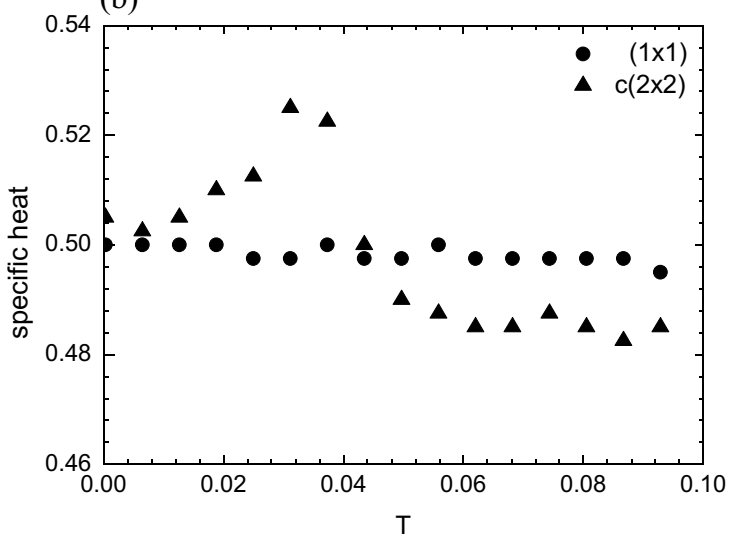

Figure 2 - Temperature dependence of the structure factor peak $S\left(k_{o}\right)$ (a) and the specific heat (b) for the $(1 \times 1)$ and $c(2 \times 2) C$ phases. $k_{o}$ is the wave vector of the corresponding ordered structures.

Figure 2 shows the behavior of the structure factor peak $S\left(k_{o}\right)$ and specific heat for increasing temperatures for the $\mathrm{C}$ phases $(1 \times 1)$ and $\mathrm{c}(2 \times 2)$, where $k_{o}$ denotes the corresponding wave vectors. For the $(1 \times 1)$ phase, $S\left(k_{o}\right)$ decreases and broadens but does not disappear at the highest temperature where a liquid-like phase is expected. Also, there is no peak in the speci- fic heat. This indicates that the $(1 \times 1)$ phase does not melt into a liquid-like phase via a phase transition. Instead, there is a smooth crossover from a low temperature highly ordered phase to a high temperature phase where the pinning potential still induces some order in the adsorbed layer. This behavior is expected on theoretical grounds $[1,8]$ since in this case the pinning potential has the same symmetry as the adsorbed layer and acts as a constant external field on the displacement order parameter.

On the other hand, for the $\mathrm{IC}$ phase and the $\mathrm{c}(2 \times 2) \mathrm{C}$ phase, $S\left(k_{o}\right)$ drops sharply near the temperature where there is a peak in the specific heat and should correspond to the melting of the structure into a liquid-like phase. There are also C-IC transitions from the $c(2 \times 2)$ to $I C$ phases and from $c(2 \times 2)$ to $(1 \times 1)$ phases which can be identified by the change in the peak patterns in the structure factor [4]. The approximate phase diagrams obtained near the $(1 \times 1)$ and $c(2 \times 2) C$ phases as a function of misfit and temperature are shown in Figure 3 . A striking feature of the phase diagram is its topology, which is strongly dependent on the type of $\mathrm{C}$ phase. This agrees with theoretical predictions from simplified models $[1,8]$.

In summary, we have studied the phase diagram of an adsorbed monolayer by MC simulations of the PFC model with external pinning introduced recently [4]. We have considered the effects of thermal fluctuations and melting in the $\mathrm{C}-\mathrm{IC}$ transition near the $(1 \times 1) \mathrm{C}$ phase and also at the additional $\mathrm{C}(2 \times 2) \mathrm{C}$ phase. The model and $\mathrm{MC}$ methods employed here [9] can be used to study more specific adsorbed systems by adjusting the parameters of the model to match the experimental structure factor of the layer $[5,6]$ and choosing a suitable pinning potential.

\section{ACKNOWLEDGMENTS}

Work supported by FAPESP (proc. 07/08492-9) (E.G.), a joint fund under EU STREP 016447 MagDot and NSF (DMR-0502737), the Academy of Finland (C.V.A., T.A.N.) and NSF (DMR-0413062) (K.R.E.).

\section{REFERENCES}

[1] PATRYKIEJEW A \& SOKOLOWSKI S. 2007. Phys. Rev. Lett., 99: 156101. PATRYKIEJEW A, SOKOLOWSKI S \& BINDER K. 2000. Surf. Sci. Rep., 37: 207. PERSSON B. 1992. ibid., 15: 1.

[2] HUPALO M, SCHMALIAN J \& TRINGIDES MC. 2003. Phys. Rev. Lett., 90: 216106.

[3] LU Y, PRZYBYLSKI M, TUSHIN O, WANG WH, BARTHEL J, GRANATO E, YING SC \& ALA-NISSILA T. 2005. Phys. Rev. Lett., 94: 146105. 

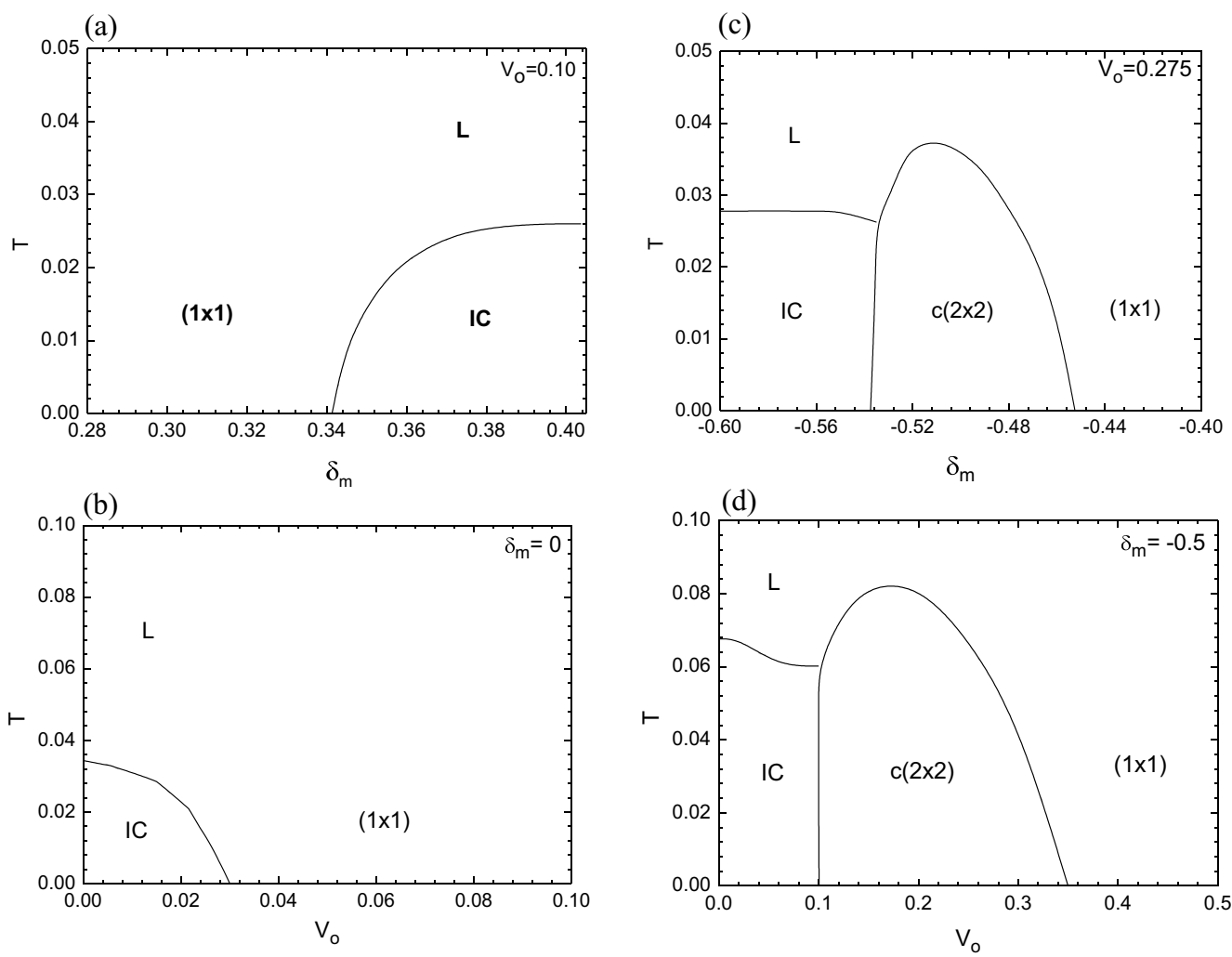

Figure 3 - Phase diagrams near the $(1 \times 1)$ and $c(2 \times 2)$ phases. (a) and (c) at fixed pinning strength $V_{o}$, (b) and (d) at fixed misfit $\delta m$. $\mathrm{L}$ denotes a liquid-like phase. The joining of the transition lines in (c) and (d) was not determined accurately.

[4] ACHIM CV, KARTTUNEN M, ELDER KR, GRANATO E, ALA-NISSILA T \& YING SC. 2006. Phys. Rev. E, 74: 021104.

[5] ELDER KR, KATAKOWSKI M, HAATAJA M \& GRANT M. 2002. Phys. Rev. Lett., 88: 245701.

[6] ELDER KR \& GRANT M. 2004. Phys. Rev. E, 70: 051605.
[7] ELDER KR, PROVATAS N, BERRY J, STEFANOVIC P \& GRANT M. 2007. Phys. Rev. B, 75: 064107.

[8] HALDANE FDM, BAK P \& BOHR T. 1983. Phys. Rev. B, 28: 2743.

[9] RAMOS JAP, GRANATO E, ACHIM CV, YING SC, ELDER KR \& ALANISSILA T. 2008. Phys. Rev. E, 78: 031109. 